\title{
Impacts and Implications of Risk Based Monitoring: A CRO Perspective
}

\section{Prashant A. Pandya*}

Department of GM-Program Management, CRS, Clinical Development, Navi Mumbai, India

\begin{abstract}
During the past decades, the complexity of clinical trials has grown dramatically due to geographic dispersion, site related issues, treatment choices, standard of care and regulatory uncertainty. The uncertainty have created opportunity for the Risk Based Monitoring (RBM) / centralize monitoring due to use of electronic system, changes in statistical assessment, improvement in clinical trial documents. RBM has emerged as the future of clinical development. This approach is supported by the US-FDA, European Medicines Agency (EMA) and several other regulatory agencies.

Fabrication of data, fraud, data distribution errors and other data anomalies that can be readily found by riskbased monitoring policies and procedures. RBM improves quality and efficiency of sponsor to oversight clinical sites and help to save significant cost. It helps to quickly identify signals that could affect quality and operational performance. It is concluded that, efficient planning lays the foundation of an effective risk-based monitoring strategy.
\end{abstract}

\section{Introduction}

The future of clinical trial monitoring is at a crossroads. Traditional monitoring is still dominating the market but risk-based monitoring has emerged as the effective tools to manage global clinical trials without compromising ethics, patient safety, quality and regulatory framework. RBM facilitate more efficient clinical trial conduct with enhance focus on quality and ethics related to patient safety. Early identification of risk helps to mitigate it with continuous monitoring throughout study life cycle [1].

\section{Monitoring in Clinical Trials}

Monitoring refers to oversee the conduct of, and reporting of data from, clinical investigations, including supervision of study site staff and third-party contractors.

Key job in monitoring is to do source data verification, site process and procedures, communication with site team members and ensure accuracy of data submitted to the regulatory authorities. It is quality control tools for the sponsor to judge whether the activities are being carried out as per approved protocols/ SOPs and regulatory norms or not. FDA recommends a quality risk management approach to clinical trials [2].

\section{Current monitoring practices}

A range of different practices has been used to monitor the conduct of clinical trials across globe. These practices differ in intensity, methodology, focus and centralized monitoring activity.

- Monitoring to high risk sites based on central monitoring findings i.e Targeted monitoring visit

- Onsite visit (By clinical research associates (CRA) or sponsor representatives) as per defined monitoring plan/ or developed as per protocol visits. For major efficacy trials, Onsite monitoring visit is preferred practice followed by most of the companies. Typically, CRAs visit site at approx. 4 to 8 -week intervals to perform $100 \%$ source data verification. It's one of preferred way to meet sponsor obligations set by FDA [2,3].

\section{Risk based monitoring}

It is "strategic" monitoring based on technologically-enabled, riskbased algorithms that focus monitoring resources on the locations and activities where they are most needed.
In 2011 the U.S. Food and Drug Administration, the European Medicines Agency and the United Kingdom's Medicines and Healthcare Products Regulatory Agency issued papers intended to "open up the discussion on approaches to clinical trials and to new thinking, in order to facilitate the development of proportionate clinical trial processes".

In August 2013 the FDA issued final guidance on the subject emphatically noting, "There is a growing consensus that risk-based approaches to monitoring, focused on risks to the most critical data elements and processes necessary to achieve study objectives, are more likely than routine visits to all clinical sites and $100 \%$ data verification to ensure subject protection and overall study quality." For e.g. incorporation of centralized monitoring practices, where appropriate, should improve a sponsor's ability to ensure the quality of clinical trial data $[1-4]$

FDA encourage to develop robust monitoring plan at the start of clinical trial which address the data quality challenges. In RBM, approach is to develop more focus and vigilant plan to mitigate likely risks to the data quality and monitor processes that linked to human subjects and ethics by protecting data integrity. In addition, centralize data checks helps to prioritizing site visits and improve the effectiveness.

\section{There are several advantages of Risk based monitoring:}

- Early identification of problems so that they can be remedied quickly, protecting patients and preserving the overall integrity of the study.

- Holistically identifies risk of failure

- Delivers fit for purpose quality -flexible to study and regulatory need.

*Corresponding author: Prashant A Pandya, Department of GM-Program Management, CRS, Clinical Development, Navi Mumbai, India, Tel: +91-9967017172; E-mail:drpandya18@gmail.com

Received January 19, 2018; Accepted January 22, 2018; Published January 27, 2018

Citation: Pandya PA (2018) Impacts and Implications of Risk Based Monitoring: A CRO Perspective. J Bioequiv Availab 10: e83. doi:10.4172/jbb.10000e83

Copyright: ( $) 2018$ Pandya PA. This is an open-access article distributed under the terms of the Creative Commons Attribution License, which permits unrestricted use, distribution, and reproduction in any medium, provided the original author and source are credited. 
Citation: Pandya PA (2018) Impacts and Implications of Risk Based Monitoring: A CRO Perspective. J Bioequiv Availab 10: e83. doi:10.4172/ jbb.10000e83

- Improves the efficiency of Clinical Research Associates (CRAs), as they concentrate on the sites that need help and allow competent sites to proceed without unnecessary interference.

- Dramatically improves the reliability and verifiability of study data, avoiding unpleasant surprises upon regulators' review.

- It has the potential to reduce overall monitoring costs in most studies.

Challenges in Risk Based Monitoring (RBM)- a Cro Perspective [1-8]: Industry is still in the transition phase and perplex towards the adoption of Risk Based Monitoring as the process is still evolving. Below are the key implementation challenges:

- Failure in the planning and execution of any activity during risk-based monitoring may raise the concern over whole clinical data generated in the study.

- Maintaining quality standard across various therapeutic areas.

- It is difficult for most of CROs to implement recommended regulatory changes in Risk based monitoring process and procedures in defined time period.

- Lack of internal knowledge, system, and procedure is one of key hurdles during implementation of RBM.

- Top management support to replace traditional monitoring system considering risk/ rewards.

- Implementation cost

- Ensuring patient safety, data integrity \& regulatory compliance

- Variable needs and priorities from different sponsors

- Expectation-setting with sites.

- Managing monitor support expectations
- Perform change and resource management

\section{Conclusion}

In spite of support from various regulatory agency like FDA, EMEA, implementation of the Risk Based Monitoring is still less across industry. The RBM policies and procedures bring as much as $20-30 \%$ cost reduction over tradition trial execution approaches with greater efficiency and more predictable outcome at much lower costs. By focusing on key data points and sites requires highest scrutiny help for efficient management of clinical trial.

It is important to create various trigger/ alert (e.g. Lab parameters, study Arm, safety signals, study drug administration, visit schedule) based on protocol design at the start of study to identify risk throughout execution period. It is concluded that, efficient planning lays the foundation of an effective risk-based monitoring strategy.

\section{References}

1. European Medicines Agency (2011) Reflection paper on Risk Based Quality Management in Clinical Trials.

2. Guidance for Industry: Oversight of Clinical Investigations - a risk-based approach to monitoring. US FDA (2013).

3. TransCelerate BioPharma Inc (2013) Position paper: Risk-Based Monitoring Methodology.

4. Nielsen E, Hyder D, Deng C (2014) A data-driven approach to riskbased source data verification. Ther Innovat Regulat Sci 48: 173-180.

5. Pogue JM, Devereaux PJ, Thorlund K, Yusuf S (2013) Central statistical monitoring: detecting fraud in clinical trials. Clin Trials 10: 225-235.

6. Pandya P (2017) Managing Complex R\&D Projects- Strategies from Project Management Perspectives. J. Pharm. Sci 1: 113-117.

7. Richard C (2014) Exploring the challenges, impacts and implications of riskbased monitoring. Clin Invest 4: 785-789.

8. Pandya PA (2017) Managing Pharmaceuticals and Life Sciences Projects on a Global Scale: A Project Management Perspective. J Bioanal Biomed 9: 169-172. 